\title{
Photometry with a periodic grid
}

\section{Results for J2 Europa and S6 Titan ${ }^{\star}$}

\author{
D. Hestroffer ${ }^{\star \star}$ \\ IMCCE, UMR CNRS 8028, Paris Observatory, 77 Av. Denfert Rochereau 75014 Paris, France
}

Received 20 December 2002 / Accepted 19 February 2003

\begin{abstract}
The Hipparcos satellite successfully gathered astrometric and photometric information for a few solar system objects including natural satellites. In contrast to the case for asteroids, the Hipparcos main-mission does not provide the photometry - in the conventional sense - of planetary satellites. Nevertheless, valuable photometric results can be obtained, such as a modelling of the centre-to-limb darkening of the bodies. We present in this paper results obtained for the photometry with a periodic grid of the satellites J2 Europa and S6 Titan from the Hipparcos main mission and within the FAST data-reduction Consortium. The Minnaert parameter of S6 Titan is found to be $k \sim 0.90 \pm 0.02$, and for J2 Europa, $k \sim 0.58 \pm 0.01$. Brightness variations with orbital phase are also given for Europa.
\end{abstract}

Key words. planets and satellites: general - planets and satellites: individual: J2 Europa, S6 Titan

\section{Introduction}

The centre-to-limb darkening or brightness distribution of celestial objects is an important parameter since it significantly affects the determination of fundamental parameters such as the radius of a star with stellar interferometry, or the position of a solar system object showing phase. The systematic phase effect for the asteroids observed by Hipparcos, although small, has a non-negligible effect on the determination of the dynamical reference frame (Söderhjelm \& Lindegren 1982). For Galilean satellites the effect is greater and has to be accounted for to enable the determination of their motion (Mallama 1993) and/or analysis of mutual event lightcurves (Descamps 1994). Introducing the non-unit vector $\boldsymbol{k}=\boldsymbol{u} \times(\boldsymbol{s} \times \boldsymbol{u})$, where $\boldsymbol{u}$ and $s$ are respectively the planeto-centric direction of the Earth and the Sun, one finds that the photocentre displacement (in the plane-of-sky view) for a spherical object of diameter $\rho$ is (Lindegren 1987):

$\Delta \boldsymbol{p}=\frac{C(\alpha)}{2 \sin \alpha} \rho \boldsymbol{k}$

where $C(\alpha)$ is a function of the solar phase angle. We have $C(0)=0$ for an observation at opposition and a homogeneous brightness distribution. At the quadratures, this displacement may vary by a large amount depending on the actual scattering properties of the planet. One finds for a uniformly

\footnotetext{
$\star$ Based on data from the Hipparcos astrometry satellite.

$\star \star$ e-mail: hestro@imcce.fr
}

bright object (U) and a scattering following Lambert's law (L), (Lindegren 1977; Hestroffer 1998):

$$
C(\alpha)=\left\{\begin{array}{l}
\frac{8}{3 \pi} \sin ^{2} \alpha / 2 \\
\text { or } \\
\frac{3 \pi}{16} \frac{1+\cos \alpha}{\sin \alpha+(\pi-\alpha) \cos \alpha} \sin \alpha
\end{array}\right.
$$

yielding a displacement five times bigger (at $\alpha \simeq 20^{\circ}$ ) for a surface of diffuse scattering than for a uniformly bright disc. For observations of Europa - at smaller phase angle, $\alpha \simeq 10^{\circ}$ - this ratio is larger, yielding a difference in the photocentre displacement of 0.013 arcsec, i.e. similar to the precision of the Hipparcos astrometric observations of that object. In the past decades other laws have been derived for atmosphereless solar system bodies; these are empirical (e.g. Minnaert 1961; Buratti \& Veverka 1983), or deduced from radiative transfer theories (Lumme \& Bowell 1981a; Hapke 1981).

With the increasing accuracies of astrometric observations (ground-based, Hipparcos, GAIA, ...), much effort has been devoted to better knowledge or modelling of the brightness distribution of solar system objects (e.g. Morando \& Lindegren 1989; Descamps 1994; Hestroffer 1998). This provides a better evaluation of the photocentre displacement (the shift of the photocentre relative to the geometric or gravity centre). Results on the brightness distribution have been obtained from speckle interferometry (McCarthy et al. 1994), analysis of the magnitude-phase curves for atmosphereless bodies (Lumme \& Bowell 1981b; Domingue \& Hapke 1989) or from analysis of lightcurves (Kaasalainen \& Torppa 2001); in particular for natural satellites from Voyager or Pioneer observations 
(Buratti \& Veverka 1983; Buratti 1984; Tomasko \& Smith 1982), or from mutual events (Descamps et al. 1992).

It has been shown in a previous paper (Hestroffer \& Mignard 1997b) that observation with a periodic grid provides the parameters of a simple centre-to-limb darkening model. The present paper gives the results obtained with this method from Hipparcos observations of natural satellites. First a general presentation of Hipparcos observations is made, next the reduction and model are developed. The application to the observations of J2 Europa and S6 Titan is given in the last sections.

\section{Hipparcos observations}

The Hipparcos satellite scanned in a regular manner the whole sky during approximately a three year interval (from November 1989 to March 1993), providing observations spread over 37 months. Due to Hipparcos' particular scanning law, observation of solar system objects only occurred around the quadratures. Thus planetary satellites of Jupiter show a phase of $\approx 10^{\circ}$, while the phase for the satellites of Saturn is $\approx 5^{\circ}$. Only objects brighter than $V \approx 12.5$ and smaller than $\approx 1^{\prime \prime}$ were observed within the main mission. This limitation in size essentially occurs from the astrometry requirement, hence 48 minor planets and three planetary satellites were added to the list of the Input Catalogue stars. The satellites are J2 Europa, S6 Titan and S8 Iapetus; the last was added later to the observing program. Unfortunately Hipparcos provides only very few useful observations of Iapetus (consisting of six transits), and moreover Iapetus is considerably smaller than the two other satellites. For these reasons the data analysis for Iapetus is less interesting and is not included in the present work, but for the sake of completeness the data can be found at the end of the paper. The observations are neither uniformly distributed in time nor over the orbit of the satellite around its gravitating planet. Moreover only observations made at a separation greater than 50 arcsec were retained.

The $0^{\circ} 9 \times 0^{\circ} 9$ field of view of the telescope scans the sky at a velocity of $168.75^{\prime \prime} / \mathrm{s}$. During a transit of a star, the incoming light is modulated by a periodic grid of period $s=1.2074^{\prime \prime}$. The fundamental periodic signal can usefully be expanded in a second order Fourier series as:

$$
\begin{aligned}
S(t)=I+B & +I M \cos (\omega t+\varphi) \\
& +I N \cos (2 \omega t+\psi)
\end{aligned}
$$

where $I$ is the total intensity, $B$ the un-modulated background, $M$ and $N$ are the modulation coefficients. The phase offsets $\varphi$ and $\psi$ are mainly of interest for the astrometry. The modulation coefficients for a point-like source have been calibrated for the whole mission, then $M_{0}$ and $N_{0}$ are reference modulation coefficients for a star. For a resolved object, larger than approximately $0^{\prime \prime} 1$ within Hipparcos, the amplitudes of the modulation are degraded (Morando 1987; Lindegren 1987; Morando \& Lindegren 1989) which translates into the fact that $M / M_{0}<1$ and $N / N_{0}<1$.

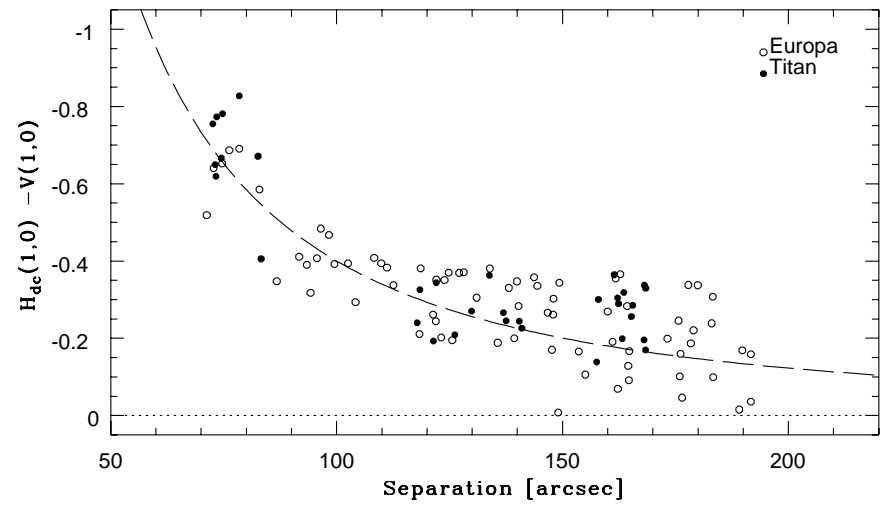

Fig. 1. Influence of the major planet disturbing stray light versus separation. It is given for the measures of the magnitude $H_{\mathrm{dc}}(1,0)-V(1,0)$ on Europa and Titan. The dashed line is a rough fit which shows a systematic error (the residual should normally be centred around zero). This stray light however does not affect the modulation of the signal, and hence the quantity $H_{\mathrm{ac}}$ is not corrupted.

Once the sky background has been removed, the apparent magnitude is naturally given in the Hipparcos photometric system $H p$ by:

$H_{\mathrm{dc}}=-2.5 \log I / I_{\mathrm{ref}}$

where $I_{\text {ref }}$ is the reference intensity of the magnitude scale. Since the modulation amplitude is proportional to the total intensity, a second magnitude estimator was constructed as:

$H_{\mathrm{ac}}=-2.5 \log \frac{I}{I_{\mathrm{ref}}} \frac{M M_{0}+N N_{0}}{M_{0}^{2}+N_{0}^{2}}$.

For a point-like source, the two estimators have the same expectation, $E\left(H_{\mathrm{ac}}\right)=E\left(H_{\mathrm{dc}}\right)$, while for planetary satellites the $H_{\mathrm{ac}}$ estimator is biased and we have:

$\Delta H=H_{\mathrm{ac}}-H_{\mathrm{dc}}=-2.5 \log \frac{M M_{0}+N N_{0}}{M_{0}^{2}+N_{0}^{2}}>0$

which is independent of the object's apparent magnitude.

While the $H_{\mathrm{ac}}$ quantity is not perturbed by stray or parasitic light of a very extended source (because the background mainly is increased by a constant amount), the observed magnitude $H_{\mathrm{dc}}$ for the planetary satellites is significantly corrupted. Figure 1 shows the residuals on the absolute magnitudes, i.e. the measured $H_{\mathrm{dc}}$ magnitude corrected to one astronomical unit and zero phase angle. Stray light from Jupiter or Saturn, although strongly attenuated at large separation, still corrupts the $H_{\mathrm{dc}}$ magnitudes that were measured by Hipparcos by about $0.1 \mathrm{mag}$. For this reason, no conventional magnitude could be determined for the Hipparcos mission planetary satellites, and the $H_{\mathrm{dc}}$ magnitudes used in Fig. 1 were hence not published in the Hipparcos catalogue solar system objects annex. We will thus evaluate the magnitude bias from the observed quantity $H_{\mathrm{ac}}$ and calculated value of $H_{\mathrm{dc}}$. The $H_{\mathrm{ac}}$ estimator is of lower accuracy than $H_{\mathrm{dc}}$, its precision also decreases with the object's brightness. The average precision on $H_{\mathrm{ac}}$ is 0.04 for Titan and 0.015 for Europa. 
Table 1. Values used in the reduction of S6 Titan and J2 Europa.

\begin{tabular}{lccccc}
\hline \hline & $R[\mathrm{~km}]$ & $V(1,0)$ & $B-V$ & $H p-V$ & $p(\alpha)$ \\
\hline S6 Titan & 2847 & -1.28 & 1.28 & 0.161 & $0.004 \alpha$ \\
J2 Europa & 1562 & -1.41 & 0.88 & 0.156 & $\left\{\begin{array}{l}0.0114 \alpha, \theta<180^{\circ} \\
0.0164 \alpha, \theta>180^{\circ}\end{array}\right.$ \\
\hline
\end{tabular}

\section{Model and reduction}

Since the magnitude $H_{\mathrm{dc}}$ is not preserved, one calculates the apparent brightness from the absolute magnitude $V(1,0)$, the distances to the Sun $r$ and to the Hipparcos satellite $\Delta$, and the solar phase angle $\alpha$ from:

$$
\begin{aligned}
\tilde{H}_{\mathrm{dc}} \equiv V(1,0) & +5 \log r \Delta+p(\alpha) \\
& +\sum_{m=1}^{4} a_{m}(B-V)^{m}
\end{aligned}
$$

where $p(\alpha)$ is a linear function of the phase accounting for the shadowed area and depending on the observed object. Observations of Europa occur at a phase angle great enough to ensure that there is no opposition effect. Here the transformation from the Johnson $V$-band magnitude to the Hipparcos system $H p$ only takes into account the $B-V$ colour index. From now on the "observed" bias will be $\Delta H=H_{\mathrm{ac}}-\tilde{H}_{\mathrm{dc}}$. Correction for solar phase and colour index are taken from the Astronomical Almanac (Franklin \& Cook 1974) and from Lockwood (1983). Basic values for the reduction in Eq. (5) are shown in Table 1; the data for the observations are given in Table 5. The accuracy of the calculated $\tilde{H}_{\mathrm{dc}}$ depends on the accuracy of the various parameters entering into Eq. (5) and is better than $0.01 \mathrm{mag}$, so that the average precision per transit on the bias $\Delta H$ is $\sim 0.01$ for Europa and $\sim 0.02$ for Titan.

The attenuation of the harmonic amplitude depends on the object's size, but also on the brightness distribution across its surface. Taking the empirical law of Minnaert (1961), this distribution can be written:

$I_{\sigma}=\mu^{k} \mu_{0}^{k-1}$

where $I_{\sigma}$ is the normalised specific intensity of surface element $\mathrm{d} \sigma, \mu_{0}$ and $\mu$ are the cosines of the angles between the surface normal and, respectively, the incident and reflected ray. The single parameter $k$ is a scalar describing the centre-to-limb darkening of the surface. For a spherical planet of apparent diameter $\rho$ with a limb darkening following Minnaert's law, it has been shown in Hestroffer \& Mignard (1997b) that this attenuation is given by the modulation function:

$$
\begin{aligned}
& x=\pi \rho / s \\
& M / M_{0}(x)=\left|{ }_{0} F_{1}\left(k+3 / 2,-x^{2} / 2\right)\right| \\
& N / N_{0}(x)=M / M_{0}(2 x)
\end{aligned}
$$

where ${ }_{0} F_{1}\left(v+1,-x^{2} / 2\right)=\Gamma(v+1) \frac{J_{v}(x)}{(x / 2)^{v}}$ is the hypergeometric function, $s=1.2074^{\prime \prime}$ is the Hipparcos grid-step and $x$ is the spatial frequency. This yields from Eq. (4) the theoretical value of the bias for a spherical object seen at opposition. Under these hypotheses, the case $k=1 / 2$ corresponds to a uniformly bright disc (which in general does not follow the reciprocity principle), while $k=1$ corresponds to the pronounced centre-to-limb

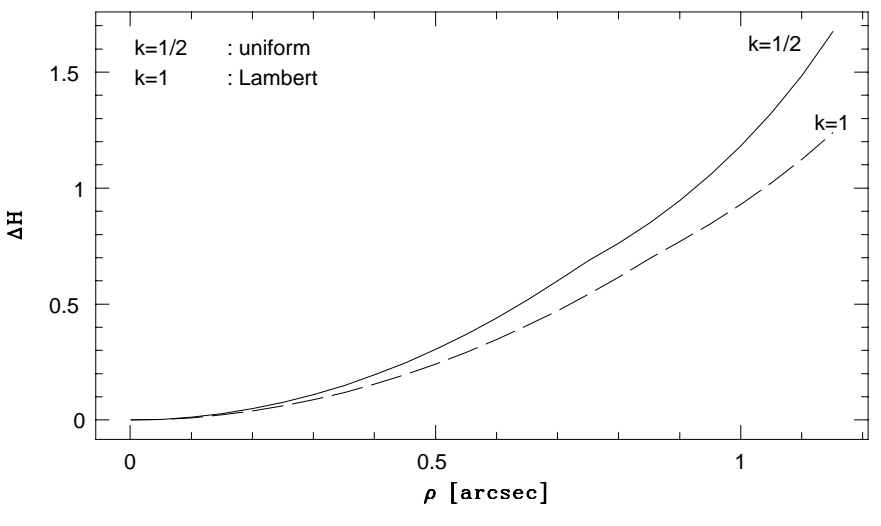

Fig. 2. Difference between the two magnitude estimators as a function of apparent diameter, for two particular cases of Minnaert's law and a spherical object seen at opposition.

darkening of the Lambertian disc. Corresponding graphs for the bias are shown in Fig. 2; the larger the coefficient $k$, the greater is the centre-to-limb darkening, yielding an apparently more point-like source and thus a smaller value of $\Delta H$. Equation (6) gives an approximation to the second order in $\alpha$, and since the phase is relatively small, improvement of the modelling will be given as small corrective terms (Hestroffer \& Mignard 1997a). The ratio of the modulation coefficients is evaluated for each point by numerical integration for an average value $\bar{k}$ of the centre-to-limb darkening. The corrective term thus depends on the apparent diameter, the solar phase angle and the scanning direction and reach its maximal value when the scan is performed in the direction nearest to the intensity equator. For Titan this correction appears to be negligible $(\lessgtr 0.002)$, and for Europa it remains small $(\lessgtr 0.015$, i.e. about $2 \%)$.

Fitting the observed magnitude bias with this model yields - for a given diameter and photometric parameters - Minnaert's parameter $k$. The accuracy of the limb-darkening parameter depends on the number of available observations and the associated error, and we have the conservative values $\sigma(k) \simeq 0.01$ for Europa, and $\sigma(k) \simeq 0.02$ for Titan.

\section{Europa}

The Galilean satellite Europa is known to have two significantly different hemispheres. The leading one, visible at eastern elongation (EE), is bright and covered with ice. In contrast, the trailing hemisphere visible at western elongation (WE) is dark and the ice is still covered by dust. Also the brightness distributions across the surface are different. In general, dark dusty surfaces appear uniform and flat, while bright icy surfaces have a more pronounced centre-to-limb darkening. Although variation of magnitude with orbital phase is a well-known phenomenon for the Galilean satellites (e.g. Morrison et al. 1974; Millis \& Thompson 1975), it depends on the solar phase angle, the subSolar point and sub-Earth point coordinates, the photometric passband used, and at least for Europa, is far from being a simple function. Its modelling in the Hipparcos photometric band would be less accurate than the other transformations entering in the reduction, thus the observations are not corrected for this effect. Hence the bias $\Delta H$ depends not only on the apparent 

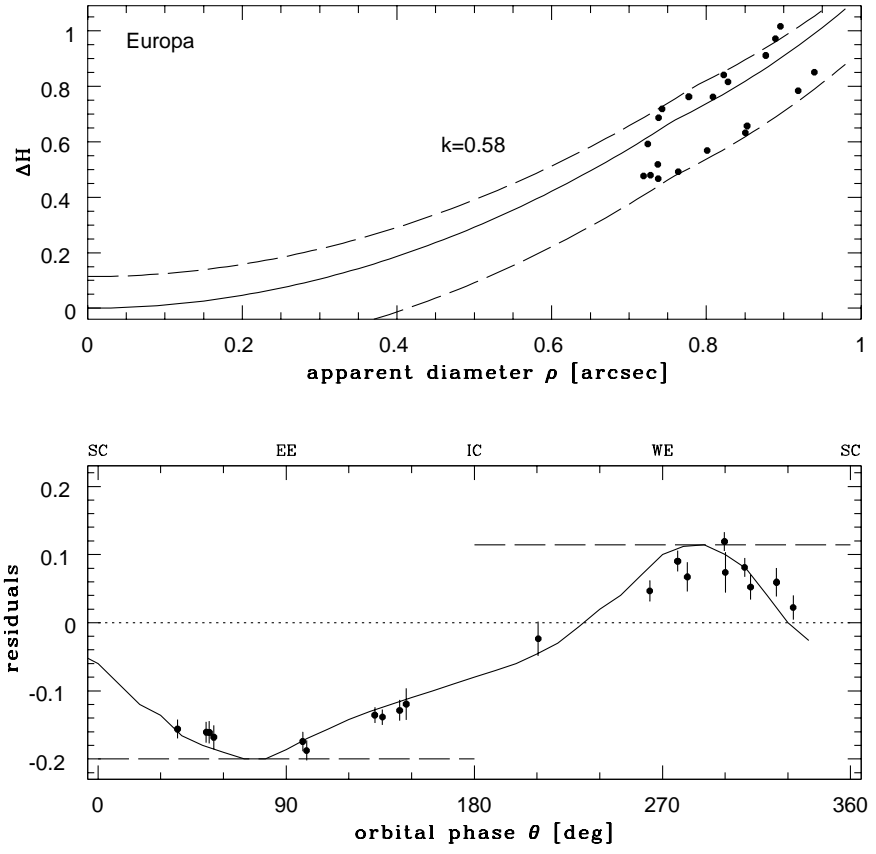

Fig. 3. Magnitude bias $\Delta H$ versus apparent diameter for Hipparcos observations of J2 Europa (top). Each point corresponds to a mean of successive transits of the planetary satellite across the field of view. The dispersion is a consequence of the variation of brightness with orbital phase. Dashed curves correspond to the bias for minimal and maximal brightness. The residuals (bottom) are calculated from light scattering following Minnaert's law with $k=0.58$. Each normal point is corrected for the effect of solar phase on the modulation function. The solid line is an independent empirical curve (see text). Filled circles correspond to observations at angular diameters larger than $0.87^{\prime \prime}$.

diameter and the limb-darkening parameter, but also on this periodic variation.

Figure 3 shows the bias as a function of the apparent diameter and the residuals obtained with a Minnaert parameter of $k=0.58$. To compare, the residuals are given as a function of the orbital phase angle $\theta$ (projected in the plane of the mean equator J2000) together with a fit to ground-based observations in the $V$ band (Morrison \& Morrison 1977). The empirical curve represents the magnitude corrected for dependence on phase $V\left(1, \alpha=6^{\circ}\right)$, and corrected for an offset between the value $V(1,0)=-1.41$ used here and the equivalent value $V^{\prime}(1,0)=-1.46$ of Morrison \& Morrison (1977). Interestingly, shifting this empirical curve by about $10^{\circ}$ for the orbital phase would provide a better fit to the Eastern data. No satisfactory explanation for this possible shift can be given here; we note however that the range in solar phase angle is slightly different between the Hipparcos $\left(8-11^{\circ}\right)$ and the ground-based observations $\left(0-11^{\circ}\right)$. Moreover, the latitude of the sub-Earth point is different between the two data sets. Last, it is not obvious if the empirical lightcurve is corrected for light-time or plane projection. Nevertheless, referring to Table 2, the values for the observed maximum $\left(\theta_{\max } \sim 85\right)$ and minimum $\left(\theta_{\min } \sim 295\right)$ magnitude of Europa are larger than the results of Morrison et al. (1974), Blanco \& Catalano (1974), but in better agreement with Johnson (1971), Millis \& Thompson (1975) and
Table 2. Longitudes in degrees of $V_{\max }$ and $V_{\min }$ for Europa.

\begin{tabular}{rcl}
\hline \hline$\theta_{\max }$ & $\theta_{\min }$ & \multicolumn{1}{c}{ Reference } \\
\hline 100 & 280 & Harris (1961) \\
90 & 300 & Johnson (1971) \\
45 & 280 & Blanco \& Catalano (1974) \\
80 & 280 & Morrison et al. (1974) \\
$\sim 90$ & $\sim 290$ & Millis \& Thompson (1975) \\
$75^{a}$ & $285^{a}$ & Morrison \& Morrison (1977) \\
95 & 295 & Domingue et al. (1991) \\
85 & 295 & Present work \\
\hline
\end{tabular}

${ }^{a}$ Synthetic data.

Domingue et al. (1991) who consider observations from 1976 to 1989.

Any discrepancy in the orbital longitudes of maximal and minimal brightness has however no consequence for the general trend of the magnitude bias and hence the determination of the Minnaert parameter. One finds a good agreement for the amplitude with the results of Morrison et al. (1974), Morrison \& Morrison (1977), Millis \& Thompson (1975) and Domingue et al. (1991). In particular, the amplitude in $B-V, b-y$ and $v-y$ are small enough so that no further correction is required, hence the amplitude and asymmetry of the light curves between the two different photometric systems are almost similar. The mean value of the parameter $k$ is acceptable for the majority of the data. The significant scatter at western elongation is also present in the results of Morrison \& Morrison (1977). A more pronounced centre-to-limb darkening $(k=0.64)$ for part of the dark trailing hemisphere (with solar elongation around $90 \pm 5^{\circ}$ ) would reduce this scatter, but is in contradiction to the fact that dark surfaces are more likely to be less limbdarkened. On the other hand, a less pronounced centre-to-limb darkening $(k=0.5)$ for the trailing hemisphere would also reduce this scatter, but it would also yield a smaller brightness amplitude (0.28), in agreement with Domingue et al. (1991). Due to the complexity of the reduction and the various crosscorrelations between the corrective terms and parameters such as solar phase angle, separation or orbital phase, no satisfactory explanation can be given here. The availability of the observed (non-corrupted) magnitudes - and hence the actual lightcurve - would have helped to put into evidence any variation of the limb-darkening parameter with the orbital phase.

\section{Titan}

In contrast to Europa, the bright Saturnian satellite Titan has formed and retained a major atmosphere. It is thus expected to show a pronounced centre-to-limb darkening. The atmosphere is very opaque for the great majority of wavelengths and there is no significant brightness variation with orbital phase in the visible light (Noland et al. 1974).

Figure 4 shows the bias as a function of the apparent diameter, and the residuals obtained with a model $k=0.9$ close to the value obtained from Pioneer data by Smith (1980). Observations around epoch $J D=2448936$ show a strong 

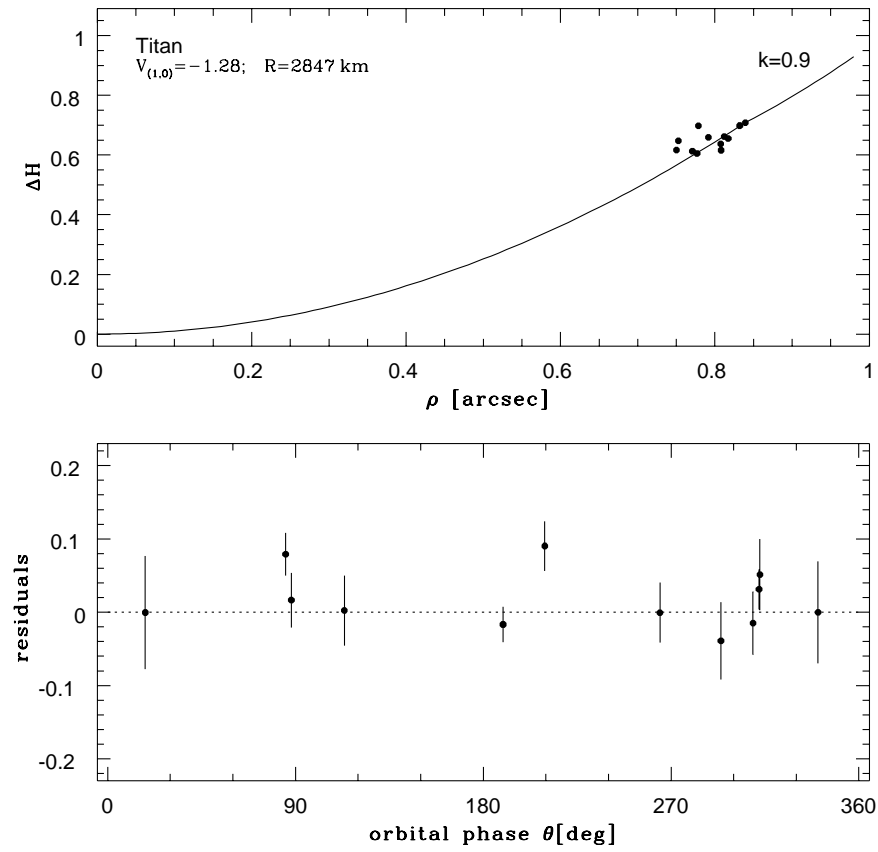

Fig. 4. Magnitude bias $\Delta H$ for Hipparcos observations of S6 Titan (top). Each point corresponds a mean of successive transits of the planetary satellite across the field of view. The residuals (bottom) are calculated from light scattering following Minnaert's law with $k=0.9$, and corrected for the effect of solar phase on the modulation function.

variation in $H_{\mathrm{ac}}(\sim 0.1)$ with the field of view, typical of parasitic light in the complementary field; two suspicious data points were thus rejected. The observations around epochs $J D=2447968$ and $J D=2447989$ show larger residuals. Although Titan was close to S7 Hyperion or S5 Rhea (with a predicted separation of less than 30 or 40 arcsec) at those epochs, the attenuation of the FOV should have been sufficient to prevent such stray light.

We adopt the absolute magnitude $V(1,0)=-1.28$ from the Astronomical Almanac, in accordance with the more recent observations made in July 1993 by Karkoschka (1994). The observations are not spread over a large range of apparent diameter, thus a small change in the value of the diameter can be balanced by a small change in the Minnaert parameter $k$ leaving the residuals almost unchanged; we have roughly the linear relation $4 \delta R / R-\delta k \simeq 0$. The situation is similar to the one encountered in lunar occultation and speckle interferometry techniques. Since the radius of Titan (with its atmosphere) cannot be considered as being a well-known quantity, we let it span the range from $R=2800$ to $R=3000 \mathrm{~km}$, and find a corresponding realistic range $0.83 \leq k \leq 1.15$ for the Minnaert parameter by minimising the L1 norm of the residuals. A minimal value of the residuals is however reached for $R=2847 \mathrm{~km}$ and $k=0.90$.

\section{Discussion}

In the absence of knowledge of the actual magnitude, analysis of the Hipparcos modulated photometry data for planetary satellites is slightly more complex than that previously obtained for the asteroid (1) Ceres (Hestroffer \& Mignard 1997b,a).
The Minnaert parameter $k$ can show variation with solar phase angle and wavelength. Moreover, since the two faces of J2 Europa have different photometric behaviours, the derived value depends on the distribution of the observations versus orbital phase. Descamps et al. (1992) found $k=0.60$ at $\lambda=410 \mathrm{~nm}$ and $k=0.57$ at $\lambda=800 \mathrm{~nm}$ from analysis of ground-based observations of a mutual event on February 10, 1991 with $\alpha=4^{\circ} 5$. Analysis of Voyager 1 and Voyager 2 observations by Buratti \& Veverka (1983) yielded the average values over the different filters $k \sim 0.63$ and 0.67 for solar phase angles of $\alpha=2.9^{\circ}$ and $3.8^{\circ}$ respectively. The limb-darkening coefficients are not strongly wavelength dependent. The parameter derived in the present study, $k=0.58 \pm 0.01$, at larger phase angle is in global agreement with the previous results. The lower value may be compatible with a variation of the limb-darkening parameter with solar phase angle. In the absence of a better knowledge of the lightcurve of Europa in the given Hipparcos filter and at the given solar phase, more refined values for the trailing and leading hemisphere seem difficult to obtain. Such a limitation would not occur if the magnitude is measured simultaneously with the modulation parameters, since the bias would be independent of the actual brightness and its variation with solar or orbital phase angles.

Using the composite cloud model of Tomasko (1980) for Titan, Smith (1980) derived an average limb-darkening parameter for the small phase angle $\alpha=6^{\circ} 25$. Using his result $k \sim 0.9$ one can derive a model-dependent value of Titan's diameter for the different techniques of observation. The lunar occultation radius is revised to $R=2845 \mathrm{~km}$, the speckle radius to $R=2742 \mathrm{~km}$, and the modulation photometry radius to $R=2847 \mathrm{~km}$. The radius found here is in good agreement with the results of Smith (1980), $R=2860 \pm 20 \mathrm{~km}$, and Elliot et al. (1975), though values of $k$ "below 1 fit [their] data poorly and appear to be ruled out". Nisenson et al. (1981) found a range $2680 \leq R \leq 2900$ for $0.8 \leq k \leq 1.15$. Although a systematic offset is present, where their data yield smaller values for the diameter, there are no contradictions between our results and the speckle data. Analysis of the best resolved observations obtained from Voyager 1 gives the optical limb (defined here by the photometric inflection) at a radius of $R(\tau=0.05)=2800 \mathrm{~km}$, with a higher optical haze layer extending up to $2845 \mathrm{~km}$ (Smith et al. 1981). Because Hipparcos is unable to resolve the thin haze layer, and because we consider a brightness step function at the limb, our result is consistent with the Voyager data. Our result is in contradiction with the earlier determinations by Dollfus (1970), $R=2435 \mathrm{~km}$, and larger than the value given by Burns (1986), $R=2775 \mathrm{~km}$. Adopting the solid-surface radius $R=2575 \mathrm{~km}$ (Lindal et al. 1983; Young et al. 2002), we find that the atmosphere extends to about $h \sim 270 \mathrm{~km}$ from the surface, hence considerably less than the value of Forrest $\&$ Nicolson (1990), $h \sim 600 \mathrm{~km}$, obtained from stellar occultation data in the $R$-filter band.

On the other hand, because the Minnaert law does not fit the entire disc, Smith (1980) derived an effective value by introducing weights proportional to the corresponding fractional disc area, hence a different weighting can yield different average values of $k$. According to Tomasko \& Smith (1982), the average limb-darkening exponents of Titan in the red $(595-720 \mathrm{~nm})$ 
Table 3. Europa's limb-darkening parameter.

\begin{tabular}{cccll}
\hline \hline $\begin{array}{c}\text { Minnaert para- } \\
\text { meter } k\end{array}$ & $\begin{array}{c}\lambda \\
{[\mathrm{nm}]}\end{array}$ & $\begin{array}{c}\text { Solar phase } \\
\text { angle } \alpha\end{array}$ & \multicolumn{1}{c}{ Method } & \multicolumn{1}{c}{ Reference } \\
\hline 0.60 & $410^{c}$ & $4^{\circ} 5$ & Mutual event (ESO) & Descamps et al. (1992) \\
0.57 & $800^{c}$ & $4^{\circ} 5$ & Mutual event (Pic du Midi) & Descamps et al. (1992) \\
$0.63^{a, e}$ & $410-580^{d}$ & $2^{\circ} 9$ & Voyager 1 & Buratti \& Veverka (1983) \\
$0.67^{a, f}$ & $410-580^{d}$ & $3^{\circ} 8$ & Voyager 2 & Buratti \& Veverka (1983) \\
0.58 & $395-895^{b}$ & $8-11^{\circ}$ & Modulated photometry & Present work $(R=1562 \mathrm{~km})$ \\
\hline
\end{tabular}

${ }^{a}$ Average value on terrain type and wavelength.

${ }^{b}$ Hipparcos $H_{p}$ broad passband.

${ }^{c}$ Effective wavelength.

${ }^{d}$ The range corresponds to different filters.

${ }^{e}$ Subspacecraft longitude of $341^{\circ}$.

${ }^{f}$ Subspacecraft longitude of $33^{\circ}$.

Table 4. Titan's limb-darkening parameter.

\begin{tabular}{cccll}
\hline \hline $\begin{array}{c}\text { Minnaert } \\
\text { parameter } k\end{array}$ & Range & $\begin{array}{c}\text { Solar phase } \\
\text { angle } \alpha\end{array}$ & \multicolumn{1}{c}{ Method } & Reference \\
\hline 1 & $\geq 1^{c, d}$ & $6^{\circ} 25$ & Lunar occultation & Elliot et al. (1975) \\
0.95 & $0.8-1.15^{c}$ & $5^{\circ} 6$ & Speckle interferometry & Nisenson et al. (1981) \\
0.9 & $0.68-1.0^{a, e}$ & $6^{\circ} 25$ & Cloud model & Smith (1980) \\
$0.87^{b}$ & $0.773-0.942^{a}$ & $29^{\circ} 6$ & Voyager 1 & Sromovsky et al. (1981) \\
$0.79^{b}$ & $0.77-0.85^{a}$ & $28^{\circ} 1$ & Pioneer & Tomasko \& Smith (1982) \\
0.90 & $0.83-1.15^{c}$ & $\sim 5^{\circ} 5$ & Modulated photometry & Present work $(R=2847 \mathrm{~km})$ \\
\hline
\end{tabular}

${ }^{a}$ The range corresponds to different filters.

${ }^{b}$ Calculated mean value for the Hipparcos broad-band photometric system $H_{p}$.

${ }^{c}$ The range is given as probable values from the given technique.

${ }^{d}$ Value of $k$ yielding residuals within one standard deviation of the minimum.

${ }^{e}$ The range also corresponds to different parts of the entire disc.

and in the blue $(390-500 \mathrm{~nm})$ are $k=0.85$ and 0.77 , respectively; these are obtained from Pioneer 11 observations at a phase of $28^{\circ}$. Voyager observations at a phase angle of $29.6^{\circ}$ yield $k=0.773,0.887,0.942$ in the violet $(370-450 \mathrm{~nm})$, blue $(430-530 \mathrm{~nm})$ and green filter $(520-600 \mathrm{~nm})$, respectively (Sromovsky et al. 1981). These results are in global agreement with the probable value taken here over a broad passband and at smaller phase angle, letting us however suspect an increased limb-darkening at small solar phase in the visible domain. Analysis of mutual events of Saturnian satellites (Arlot \& Thuillot 1993; Aksnes \& Dourneau 1994; Thuillot et al. 2001) may shed some light on the actual average limb-darkening parameter at small phase.

\section{Conclusion}

Modulated photometry with a periodic grid appears to be a interesting method to derive a simple modelling of the centre-to-limb darkening of celestial objects. Results obtained from Hipparcos observations of the minor planet (1) Ceres (Hestroffer \& Mignard 1997b), and the natural satellites J2 Europa and S6 Titan have shown some of the potential of this method. The analysis of the Hipparcos observations of natural satellites shown here is more complex because the apparent magnitudes in the Hipparcos photometric system were not available. For Titan this translates, once the absolute magnitude $V(1,0)$ of the satellite is fixed, into a high correlation between the two unknowns, namely the Minnaert parameter $k$ and the radius, and a relatively large range of probable values for $k$. The solution that minimises the L1 norm of the residuals is found for $k=0.90 \pm 0.02$ and $R=2847 \mathrm{~km}$. For Europa, variation of the limb-darkening coefficient with orbital phase and solar phase angles are difficult to decorrelate from the variation of the apparent brightness. The best-fit solution is $k=0.58 \pm 0.01$ (assuming $R=1562 \mathrm{~km}$ ) over a complete orbital period and over the range in solar phase $8<\alpha<11$.

In a normal context, those limitations encountered for Europa would not appear since the analysis of the modulated photometry with a periodic grid would be done with the measured apparent brightness. On an other hand, this modulatedphotometry method is completely orthogonal to the one used, for instance, by Lumme \& Bowell (1981b), Domingue \& Hapke (1989) or Kaasalainen et al. (2002) to derive the scattering properties of solar system objects. The law of Minnaert is empirical and gives a rough estimation of the scattering properties of solar system bodies' surface or atmosphere. 
Table 5. Hipparcos observations of Europa, Titan and Iapetus, and corresponding aspect data. The date of observation is given in TDT with respect to JD 2440000.0 , the non-conventional and biased magnitude $H_{\mathrm{ac}}$ and corresponding precision are given in fields 2 and 3 . The distances to the Sun $r$ and to the Hipparcos satellite $\Delta$, the solar phase angle $\alpha$ and the projected orbital phase $\theta$ are given in fields 4 to 7 .

\begin{tabular}{|c|c|c|c|c|c|c|c|c|c|c|c|c|c|}
\hline \multicolumn{7}{|c|}{ J2 Europa } & \multicolumn{7}{|c|}{ S6 Titan } \\
\hline $\begin{array}{c}\text { Date } \\
\mathrm{JD}-2.44 \times 10^{6}\end{array}$ & $H_{\mathrm{ac}}$ & $\sigma_{H_{\mathrm{ac}}}$ & $\begin{array}{c}r \\
{[\mathrm{AU}]}\end{array}$ & $\begin{array}{c}\Delta \\
{[\mathrm{AU}]}\end{array}$ & $\begin{array}{c}\alpha \\
{[\operatorname{deg}]}\end{array}$ & $\begin{array}{c}\theta \\
{[\mathrm{deg}]}\end{array}$ & $\begin{array}{c}\text { Date } \\
\mathrm{JD}-2.44 \times 10^{6}\end{array}$ & $H_{\mathrm{ac}}$ & $\sigma_{H_{\mathrm{ac}}}$ & $\begin{array}{c}r \\
{[\mathrm{AU}]}\end{array}$ & $\begin{array}{c}\Delta \\
{[\mathrm{AU}]}\end{array}$ & $\begin{array}{c}\alpha \\
{[\mathrm{deg}]}\end{array}$ & $\begin{array}{r}\theta \\
{[\mathrm{deg}]}\end{array}$ \\
\hline 7963.82977 & 6.8980 & 0.0254 & 5.180 & 4.934 & 10.94 & 319.25 & 7967.95956 & 9.6292 & 0.0338 & 10.021 & 10.433 & 5.08 & 83.21 \\
\hline 7963.84411 & 6.9154 & 0.0191 & 5.180 & 4.935 & 10.94 & 320.72 & 7967.97389 & 9.6436 & 0.0409 & 10.021 & 10.433 & 5.08 & 83.52 \\
\hline 7963.91867 & 6.8180 & 0.0088 & 5.180 & 4.936 & 10.94 & 328.35 & 7968.13735 & 9.6204 & 0.0183 & 10.021 & 10.430 & 5.09 & 87.04 \\
\hline 7963.93298 & 6.8694 & 0.0207 & 5.180 & 4.937 & 10.94 & 329.81 & 7968.15168 & 9.6881 & 0.0198 & 10.021 & 10.430 & 5.09 & 87.35 \\
\hline 7993.15004 & 6.6492 & 0.0063 & 5.191 & 5.402 & 10.62 & 47.84 & 7989.46454 & 9.6124 & 0.0346 & 10.013 & 10.083 & 5.70 & 208.36 \\
\hline 7993.16435 & 6.6722 & 0.0124 & 5.191 & 5.402 & 10.62 & 49.26 & 7989.47886 & 9.5346 & 0.0368 & 10.013 & 10.083 & 5.70 & 208.67 \\
\hline 7993.25324 & 6.7028 & 0.0215 & 5.191 & 5.403 & 10.61 & 58.08 & 7989.55342 & 9.6854 & 0.0293 & 10.013 & 10.081 & 5.70 & 210.25 \\
\hline 8011.45755 & 6.6897 & 0.0126 & 5.194 & 5.666 & 9.41 & 99.76 & 7989.56777 & 9.6589 & 0.0219 & 10.013 & 10.081 & 5.70 & 210.56 \\
\hline 8150.64483 & 6.7835 & 0.0243 & 5.240 & 5.871 & 8.11 & 143.67 & 8144.45433 & 9.4874 & 0.0434 & 10.002 & 9.433 & 4.91 & 111.71 \\
\hline 8150.71939 & 6.8111 & 0.0189 & 5.240 & 5.870 & 8.12 & 151.20 & 8144.46864 & 9.4768 & 0.0291 & 10.002 & 9.433 & 4.91 & 112.03 \\
\hline 8194.96798 & 6.9773 & 0.0112 & 5.263 & 5.261 & 10.84 & 309.34 & 8144.54322 & 9.4864 & 0.0595 & 10.002 & 9.434 & 4.91 & 113.71 \\
\hline 8239.32128 & 6.6181 & 0.0109 & 5.272 & 4.606 & 8.45 & 126.87 & 8144.55753 & 9.4742 & 0.0540 & 10.001 & 9.434 & 4.91 & 114.03 \\
\hline 8239.39585 & 6.6188 & 0.0115 & 5.272 & 4.605 & 8.44 & 134.47 & 8144.63210 & 9.4406 & 0.0481 & 10.001 & 9.435 & 4.92 & 115.71 \\
\hline 8239.41017 & 6.6230 & 0.0094 & 5.272 & 4.605 & 8.44 & 135.91 & 8163.64994 & 9.4892 & 0.0178 & 9.994 & 9.722 & 5.61 & 188.29 \\
\hline 8363.20474 & 6.6430 & 0.0121 & 5.317 & 5.086 & 10.75 & 92.55 & 8163.66428 & 9.4163 & 0.0199 & 9.994 & 9.722 & 5.61 & 188.63 \\
\hline 8363.21908 & 6.6801 & 0.0151 & 5.317 & 5.086 & 10.75 & 94.03 & 8163.73883 & 9.4888 & 0.0374 & 9.994 & 9.723 & 5.61 & 190.34 \\
\hline 8363.29362 & 6.6538 & 0.0089 & 5.316 & 5.087 & 10.76 & 101.75 & 8163.75315 & 9.5202 & 0.0196 & 9.994 & 9.723 & 5.61 & 190.67 \\
\hline 8363.30796 & 6.6688 & 0.0117 & 5.316 & 5.087 & 10.76 & 103.23 & 8191.20833 & 9.5916 & 0.0524 & 10.000 & 10.183 & 5.56 & 87.20 \\
\hline 8393.41358 & 7.0572 & 0.0154 & 5.325 & 5.564 & 10.33 & 271.44 & 8191.28289 & 9.5191 & 0.0183 & 10.000 & 10.184 & 5.55 & 88.80 \\
\hline 8393.42791 & 7.0036 & 0.0190 & 5.325 & 5.564 & 10.33 & 272.98 & 8393.36906 & 9.5216 & 0.0441 & 9.978 & 9.608 & 5.50 & 307.46 \\
\hline 8393.51680 & 7.0680 & 0.0147 & 5.326 & 5.566 & 10.33 & 282.55 & 8393.38341 & 9.4666 & 0.0245 & 9.978 & 9.608 & 5.50 & 307.80 \\
\hline 8393.59140 & 7.0102 & 0.0142 & 5.326 & 5.568 & 10.32 & 290.54 & 8393.45795 & 9.4596 & 0.0720 & 9.978 & 9.607 & 5.49 & 309.56 \\
\hline 8393.60567 & 7.0512 & 0.0353 & 5.326 & 5.568 & 10.32 & 292.06 & 8393.47227 & 9.5159 & 0.0312 & 9.978 & 9.606 & 5.49 & 309.90 \\
\hline 8411.36583 & 7.0984 & 0.0301 & 5.332 & 5.822 & 9.12 & 289.98 & 8393.54683 & 9.3698 & 0.0368 & 9.978 & 9.605 & 5.49 & 311.68 \\
\hline 8411.38014 & 7.0669 & 0.0227 & 5.332 & 5.822 & 9.12 & 291.50 & 8410.61050 & 9.5133 & 0.0717 & 9.977 & 9.355 & 4.74 & 339.44 \\
\hline 8411.54360 & 7.0707 & 0.0241 & 5.333 & 5.826 & 9.10 & 308.70 & 8410.62485 & 9.3899 & 0.0116 & 9.977 & 9.354 & 4.74 & 339.80 \\
\hline 8411.55792 & 7.0625 & 0.0257 & 5.333 & 5.826 & 9.10 & 310.19 & 8410.71373 & 9.4712 & 0.1198 & 9.977 & 9.353 & 4.73 & 342.03 \\
\hline 8549.33448 & 6.9919 & 0.0157 & 5.366 & 5.974 & 8.03 & 205.48 & 8539.42820 & 9.5794 & 0.0806 & 9.957 & 9.667 & 5.60 & 17.81 \\
\hline 8549.34877 & 7.0002 & 0.0432 & 5.366 & 5.974 & 8.03 & 206.87 & 8539.44253 & 9.3846 & 0.0710 & 9.957 & 9.667 & 5.60 & 18.16 \\
\hline 8549.42334 & 6.9818 & 0.0096 & 5.367 & 5.973 & 8.03 & 214.17 & 8566.52804 & 9.5162 & 0.0282 & 9.944 & 10.105 & 5.61 & 263.64 \\
\hline 8549.43765 & 7.0268 & 0.0225 & 5.367 & 5.973 & 8.03 & 215.59 & 8566.54239 & 9.6069 & 0.0212 & 9.944 & 10.105 & 5.61 & 263.94 \\
\hline 8558.40064 & 6.8276 & 0.0171 & 5.375 & 5.868 & 8.84 & 40.53 & 8566.61695 & 9.5599 & 0.0572 & 9.944 & 10.107 & 5.61 & 265.46 \\
\hline 8558.41492 & 6.8202 & 0.0210 & 5.375 & 5.868 & 8.84 & 41.98 & 8566.63125 & 9.3924 & 0.0438 & 9.944 & 10.107 & 5.61 & 265.76 \\
\hline 8558.48955 & 6.7966 & 0.0128 & 5.374 & 5.867 & 8.85 & 49.73 & 8712.81732 & 9.5496 & 0.0536 & 9.925 & 10.465 & 4.71 & 311.35 \\
\hline 8558.50384 & 6.7716 & 0.0158 & 5.374 & 5.866 & 8.85 & 51.24 & 8712.83166 & 9.6224 & 0.0611 & 9.925 & 10.465 & 4.71 & 311.69 \\
\hline 8558.57842 & 6.7983 & 0.0111 & 5.374 & 5.865 & 8.86 & 59.22 & 8712.90620 & 9.5800 & 0.0242 & 9.925 & 10.464 & 4.71 & 313.48 \\
\hline 8558.59273 & 6.8304 & 0.0191 & 5.374 & 5.864 & 8.86 & 60.78 & 8712.92054 & 9.6434 & 0.0476 & 9.925 & 10.464 & 4.71 & 313.82 \\
\hline 8558.66730 & 6.8002 & 0.0102 & 5.373 & 5.863 & 8.87 & 68.98 & 8760.00098 & 9.4410 & 0.0590 & 9.914 & 9.715 & 5.77 & 293.73 \\
\hline 8558.68162 & 6.8119 & 0.0221 & 5.373 & 5.862 & 8.87 & 70.57 & 8760.01529 & 9.4363 & 0.0389 & 9.914 & 9.715 & 5.77 & 294.06 \\
\hline 8593.22785 & 6.9651 & 0.0138 & 5.385 & 5.353 & 10.56 & 331.89 & 8935.89165 & 9.6421 & 0.0360 & 9.879 & 9.916 & 5.76 & 310.77 \\
\hline 8593.24217 & 6.9949 & 0.0164 & 5.385 & 5.352 & 10.56 & 333.24 & 8935.90599 & 9.5381 & 0.0318 & 9.879 & 9.917 & 5.76 & 311.11 \\
\hline 8624.59019 & 6.9270 & 0.0139 & 5.389 & 4.867 & 9.36 & 268.88 & 8935.98055 & 9.6434 & 0.0307 & 9.879 & 9.918 & 5.76 & 312.89 \\
\hline 8624.60452 & 6.9930 & 0.0143 & 5.389 & 4.867 & 9.36 & 270.46 & 8935.99484 & 9.5006 & 0.0201 & 9.879 & 9.918 & 5.76 & 313.23 \\
\hline 8624.67909 & 6.9918 & 0.0109 & 5.390 & 4.866 & 9.35 & 278.64 & \multirow{2}{*}{\multicolumn{7}{|c|}{ S8 Iapetus }} \\
\hline 8624.69341 & 6.9746 & 0.0139 & 5.390 & 4.866 & 9.35 & 280.20 & & & & & & & \\
\hline 8624.76801 & 6.9403 & 0.0118 & 5.390 & 4.865 & 9.34 & 288.28 & & & & & & & \\
\hline 8636.58891 & 6.6592 & 0.0176 & 5.394 & 4.711 & 8.08 & 42.85 & Date & $H_{\mathrm{ac}}$ & $\sigma_{H_{\mathrm{ac}}}$ & $r$ & $\Delta$ & $\alpha$ & $\theta$ \\
\hline 8636.60325 & 6.6658 & 0.0132 & 5.394 & 4.711 & 8.08 & 44.36 & $\mathrm{JD}-2.44 \times 10^{6}$ & & & {$[\mathrm{AU}]$} & {$[\mathrm{AU}]$} & [deg] & [deg] \\
\hline 8636.67778 & 6.6466 & 0.0119 & 5.393 & 4.709 & 8.07 & 52.30 & 8760.00099 & 11.0265 & 0.0647 & 9.894 & 9.694 & 5.78 & 220.64 \\
\hline 8636.69213 & 6.6803 & 0.0173 & 5.393 & 4.709 & 8.07 & 53.86 & 8760.01530 & 11.1648 & 0.0397 & 9.894 & 9.693 & 5.78 & 220.70 \\
\hline 8636.76672 & 6.6007 & 0.0109 & 5.393 & 4.708 & 8.06 & 62.04 & 8935.89167 & 11.2251 & 0.0230 & 9.885 & 9.924 & 5.75 & 301.06 \\
\hline 8636.78104 & 6.6265 & 0.0195 & 5.392 & 4.707 & 8.06 & 63.63 & 8935.90601 & 10.9094 & 0.0750 & 9.885 & 9.924 & 5.75 & 301.12 \\
\hline 8762.90144 & 7.0225 & 0.0167 & 5.415 & 5.224 & 10.68 & 257.86 & 8935.98056 & 10.9685 & 0.0441 & 9.885 & 9.925 & 5.75 & 301.45 \\
\hline 8762.97599 & 6.9640 & 0.0122 & 5.416 & 5.226 & 10.68 & 266.18 & 8935.99486 & 11.1772 & 0.0418 & 9.885 & 9.926 & 5.75 & 301.51 \\
\hline 8762.99029 & 7.0032 & 0.0109 & 5.416 & 5.226 & 10.68 & 267.78 & & & & & & & \\
\hline 8811.31466 & 6.8496 & 0.0112 & 5.423 & 5.944 & 8.78 & 123.79 & & & & & & & \\
\hline 8811.49244 & 6.8693 & 0.0090 & 5.422 & 5.946 & 8.77 & 141.47 & & & & & & & \\
\hline 8811.50677 & 6.8844 & 0.0062 & 5.422 & 5.946 & 8.77 & 142.86 & & & & & & & \\
\hline 8946.63691 & 6.8651 & 0.0157 & 5.438 & 6.021 & 8.05 & 134.66 & & & & & & & \\
\hline 8946.65126 & 6.8919 & 0.0058 & 5.438 & 6.021 & 8.05 & 136.11 & & & & & & & \\
\hline 8946.74014 & 6.9092 & 0.0177 & 5.438 & 6.019 & 8.06 & 144.94 & & & & & & & \\
\hline 8946.81469 & 6.8996 & 0.0119 & 5.438 & 6.018 & 8.07 & 152.17 & & & & & & & \\
\hline 8946.82901 & 6.8862 & 0.0089 & 5.438 & 6.018 & 8.07 & 153.55 & & & & & & & \\
\hline 8958.96801 & 7.1089 & 0.0099 & 5.446 & 5.863 & 9.12 & 303.70 & & & & & & & \\
\hline 8959.04257 & 7.0488 & 0.0143 & 5.446 & 5.862 & 9.12 & 311.18 & & & & & & & \\
\hline 8959.05690 & 7.1614 & 0.0261 & 5.446 & 5.862 & 9.12 & 312.59 & & & & & & & \\
\hline 8959.14580 & 7.0929 & 0.0136 & 5.447 & 5.861 & 9.13 & 321.22 & & & & & & & \\
\hline 9009.71856 & 6.7202 & 0.0122 & 5.450 & 5.074 & 9.95 & 37.30 & & & & & & & \\
\hline 9009.73289 & 6.7311 & 0.0143 & 5.449 & 5.074 & 9.95 & 38.78 & & & & & & & \\
\hline 9026.39988 & 7.0041 & 0.0154 & 5.450 & 4.831 & 8.60 & 294.34 & & & & & & & \\
\hline 9026.41418 & 7.0254 & 0.0148 & 5.450 & 4.831 & 8.60 & 295.83 & & & & & & & \\
\hline 9026.48877 & 7.0283 & 0.0086 & 5.451 & 4.831 & 8.59 & 303.48 & & & & & & & \\
\hline 9026.50308 & 6.9660 & 0.0132 & 5.451 & 4.830 & 8.59 & 304.93 & & & & & & & \\
\hline
\end{tabular}


The more appropriate 2 parameter-model of Buratti \& Veverka (1983) could also be used in photometric observations with a periodic grid.

\section{References}

Aksnes, K., \& Dourneau, G. 1994, Icarus, 112, 545

Arlot, J., \& Thuillot, W. 1993, Icarus, 105, 427

Blanco, C., \& Catalano, S. 1974, A\&A, 33, 105

Buratti, B., \& Veverka, J. 1983, Icarus, 55, 93

Buratti, B. J. 1984, Icarus, 59, 392

Burns, J. A. 1986, in Some Background about Satellites, ed. J. A. Burns, \& M. S. Mathews, IAU Colloq., 77, 1

Descamps, P. 1994, A\&A, 291, 664

Descamps, P., Arlot, J. E., Thuillot, W., et al. 1992, Icarus, 100, 235

Dollfus, A. 1970, Surfaces and interiors of planets and satellites, ed. A. Dollfus (London: Academic Press), 46

Domingue, D., \& Hapke, B. 1989, Icarus, 78, 330

Domingue, D. L., Hapke, B. W., Lockwood, G. W., \& Thompson, D. T. 1991, Icarus, 90,30

Elliot, J. L., Veverka, J., \& Goguen, J. 1975, Icarus, 26, 387

Forrest, R. W., \& Nicolson, I. K. M. 1990, MNRAS, 243, 10P

Franklin, F. A., \& Cook, A. F. 1974, Icarus, 23, 355

Hapke, B. 1981, J. Geophys. Res., 86, 3039

Harris, D. L. 1961, in Planets and Satellites, ed. G. P. Kuiper, \& B. M. Middlehurst (The University of Chicago Press), 272

Hestroffer, D. 1998, A\&A, 336, 776

Hestroffer, D., \& Mignard, F. 1997a, ESA SP-402: Hipparcos - Venice '97, 402, 173

Hestroffer, D., \& Mignard, F. 1997b, A\&A, 325, 1253

Johnson, T. V. 1971, Icarus, 14, 94

Kaasalainen, M., \& Torppa, J. 2001, Icarus, 153, 24

Kaasalainen, M., Torppa, J., \& Piironen, J. 2002, Icarus, 159, 369

Karkoschka, E. 1994, Icarus, 111, 174

Lindal, G. F., Wood, G. E., Hotz, H. B., et al. 1983, Icarus, 53, 348
Lindegren, L. 1977, A\&A, 57, 55

Lindegren, L. 1987, in Third FAST Thinkshop, ed. P. L. Bernacca, \& J. Kovalevsky, 285-293

Lockwood, G. W. 1983, in Solar system photometry handbook, ed. R. M. Genet (Willmann-Bell, Inc.), Chap. 2

Lumme, K., \& Bowell, E. 1981a, AJ, 86, 1694

Lumme, K., \& Bowell, E. 1981b, AJ, 86, 1705

Mallama, A. 1993, J. Geophys. Res., 98, 18873

McCarthy, D. W., Freeman, J. D., \& Drummond, J. D. 1994, Icarus, 108,285

Millis, R. L., \& Thompson, D. T. 1975, Icarus, 26, 408

Minnaert, M. 1961, in Planets and Satellites, ed. G. P. Kuiper, \& B. M. Middlehurst (The University of Chicago Press), 213

Morando, B. 1987, in Third FAST Thinkshop, ed. P. L. Bernacca, \& J. Kovalevsky, 277-283

Morando, B., \& Lindegren, L. 1989, in The Hipparcos Mission. Prelaunch Status: The Data Reductions, Vol. 3 (ESA SP-1111), 269

Morrison, D., \& Morrison, N. D. 1977, in Planetary Satellites, ed. J. A. Burns, IAU Colloq., 28, 363

Morrison, D., Morrison, N. D., \& Lazarewicz, A. R. 1974, Icarus, 23, 399

Nisenson, P., Apt, J., Horowitz, P., \& Goody, R. 1981, AJ, 86, 1690

Noland, M., Veverka, J., Morrison, D., et al. 1974, Icarus, 23, 334

Smith, B. A., Soderblom, L., Beebe, R. F., et al. 1981, Science, 212, 163

Smith, P. H. 1980, J. Geophys. Res., 85, 5943

Söderhjelm, S., \& Lindegren, L. 1982, A\&A, 110, 156

Sromovsky, L. A., Suomi, V. E., Pollack, J. B., et al. 1981, Nature, 292, 698

Thuillot, W., Arlot, J.-E., Ruatti, C., et al. 2001, A\&A, 371, 343

Tomasko, M. G. 1980, J. Geophys. Res., 85, 5937

Tomasko, M. G., \& Smith, P. H. 1982, Icarus, 51, 65

Young, E. F., Rannou, P., McKay, C. P., Griffith, C. A., \& Noll, K. 2002, AJ, 123, 3473 